\title{
Dipeptides of Glutamate and Aspartate May be Endogenous Neuroexcitants in the Rat Hippocampal Slice ${ }^{1}$
}

\author{
JOHN BERNSTEIN, ROBERT S. FISHER, ${ }^{2}$ ROBERT ZACZEK, AND JOSEPH COYLE
}

Departments of Neurology, Psychiatry, Neuroscience, and Pharmacology, Johns Hopkins University School of Medicine, Baltimore, MD 21205

\begin{abstract}
The dipeptide $\mathrm{N}$-acetylaspartylglutamate (NAAG), and possibly the related dipeptide aspartylglutamate (AG), have been found in high concentrations in rat brain, and have been shown to bind avidly and selectively to a subset of glutamate (GLUT) receptors. Certain observations regarding GLUT and aspartate might be explained if the endogenous transmitter were a compound composed of both amino acids. We therefore examined the electrophysiological actions of NAAG and $A G$ in the rat in vitro rat hippocampal slice model.

NAAG or AG and GLUT were applied locally to cells by a dual-barrel pressure technique. Intracellular recordings from 34 CA1 pyramidal neurons showed depolarizations and conductance increases resembling evoked excitatory postsynaptic potential in $\mathbf{1 5}$ of 20 cells exposed to NAAG, and 14 of 14 exposed to AG. Many GLUT-responsive sites did not respond to $A G$, and most did not respond to NAAG. Responses to NAAG were usually too small to induce cell firing; they were best detected, therefore, by intracellular recording.

With extracellular unit recordings, GLUT was equally excitatory in stratum radiatum and pyramidale of CA1 $(N=19 ; p$ $>0.10$, one-way analysis of variance). In contrast, AG was considerably more potent $(N=21 ; p<0.01)$ in stratum radiatum. NAAG was not noted to excite cells when applied to stratum pyramidale. The region of maximal responsiveness to AG in CA1 coincided with the area of the dendritic tree receiving Schaffer collateral-commissural afferents. This spatial profile, together with other neuropharmacological evidence, support the candidacy of glutamate-containing peptides as endogenous excitatory compounds in certain pathways of hippocampus.
\end{abstract}

The acidic dipeptide $N$-acetylaspartylglutamate (NAAG) exists in high concentrations in rat brain, including hippocampus (Curatolo et al., 1965; Reichert and Fonnum, 1969; Koller et al., 1984), binds

Received May 22, 1984; Revised November 5, 1984;

Accepted November 8, 1984

${ }^{1}$ This work was supported by Teacher Investigator Development Award Grant 5 K07 NS00697-03, a grant from The Epilepsy Foundation of America The Gertrude Sergievsky Fund for Epilepsy, and contributions from Mr. and Mrs. Palmer Jones and Associates. Ms. Kerry Koller and Dr. Elis Stanley provided valuable discussions of the findings. Mr. Otto Kausch provided information on background neurochemical issues pertaining to aspartylglutamate.

${ }^{2}$ To whom all correspondence should be directed, at Department of Neurology, Johns Hopkins University School of Medicine, 600 N. Wolfe St., Baltimore, MD 21205. selectively to a subpopulation of synaptic glutamate (GLUT) receptors with an affinity greater than that of glutamate itsclf (Koller and Coyle, 1984) and is convulsant when injected into rat hippocampus (Zaczek et al., 1983). In recent work, NAAG has been found to excite cells in prepyriform cortex; it has been theorized that it may be the endogenous transmitter of the lateral olfactory tract (ffrenchMullen et al., 1985). The related dipeptide, aspartylglutamate (AG), has also recently been shown to displace GLUT strongly from forebrain GLUT receptors (Koller and Coyle, 1984); in preliminary work, an endogenous substance with chromatographic characteristics identical to those of AG has been identified in brain (Zaczek, Kausch, and Coyle, manuscript in preparation).

GLUT is a prime candidate for the endogenous transmitter at the Schaffer-commissural pathway of hippocampus (Cotman et al., 1981; Fonnum, 1984; Nadler et al., 1976). There have been certain difficulties in assigning it a definitive transmitter role. First, it has been found to be strongly excitatory in areas of hippocampus where there are no known excitatory afferents (Andersen, 1981; Schwartzkroin and Andersen, 1975; Shepherd, 1979). Second, it has proved difficult to distinguish the actions of GLUT from those of aspartate (ASP) in this pathway: both GLUT and ASP (endogenous, or exogenously supplied) are released in a $\mathrm{Ca}^{++}$-dependent manner on electrical stimulation (Cotman and Nadler, 1981; Malthe-Sorensson et al., 1979; Wieraszko and Lynch, 1979); both are taken up by the sarme high-affinity transport mechanism (Balcar and Johnston, 1972); both are demonstrable by autoradiography in nerve terminals in the same regions of stratum oriens and stratum radiatum, but not stratum pyramidale (Storm-Mathisen, 1981); and both are strongly excitatory (Coyle, 1980). Third, recent work employing desensitization techniques has brought out apparent differences between the action of the endogenous transmitter and that of GLUT (Fagni et al., 1983).

If the neurotransmitter were a peptide containing both GLUT and ASP, this might explain the observed inseparable release of GLUT and ASP from the Schaffer-commissural pathway, and also the apparent differences between effects of applied GLUT and of stimulation of afferent fibers. We have, therefore, examined the neurophysiological actions of NAAG and AG in the in vitro rat hippocampal slice (hereafter, "in vitro" will be implicit) in order to evaluate their potential candidacies as hippocampal neurotransmitters.

\section{Materials and Methods}

Experiments were performed on $400-\mu \mathrm{m}$-thick slices from hippocampi of 150- to 200-g Sprague-Dawley rats using standard techniques (Langmoen and Andersen, 1981). Single slices were transferred to a submersion-type recording chamber maintained at 30 to $32^{\circ} \mathrm{C}$. Immersion solution consisted of (mM): $\mathrm{NaCl}, 122.6 ; \mathrm{KCl}, 5.4 ; \mathrm{MgSO}_{4}, 2.0 ; \mathrm{CaCl}_{2}, 2.5 ; \mathrm{NaH}_{2} \mathrm{PO}_{4}, 1.2$; $\mathrm{NaHCO}_{3}, 26.2$; and glucose, 10. In experiments using low-calcium solutions, calcium was omitted and magnesium raised to $6 \mathrm{~mm}$. Solutions, bubbled with $95 \% \mathrm{O}_{2} / 5 \% \mathrm{CO}_{2}$, were gravity-fed through the chamber at a rate of 0.3 to $0.5 \mathrm{ml} / \mathrm{min}$.

All drugs were refrigerated when not in use. Synthetic NAAG and AG were 
donated by Dr. Kathleen Biziere (Clin-Midi, Montpelier, France). In prior work, the identity of these compounds was verified by Dr. Coyle and associates in two ways. First, upon hydrolysis both yielded equimolar concentrations of ASP and GLUT. No other amino acid was found in the hydrolysate. Second, both NAAG and AG were analyzed by fast atom bombardment mass spectrometry. Analysis of molecular weight of the ion and fragmentation patterns confirmed the identity of the compounds. We noted neither change in potency nor breakdown of these agents after 1 to 2 weeks of refrigeration. All other chemicals were purchased from Sigma.

intracellular microelectrodes, pulled to 50 to 100 megohms (at $135 \mathrm{~Hz}$ ), were filled with $3 \mathrm{M}$ potassium methyl sulfate. Bipolar stimulating electrodes were used to deliver $10-$ to $80-\mu \mathrm{sec}$ stimuli to $\mathrm{s}$. radiatum or alveus. Only neurons with resting potentials greater than $-50 \mathrm{mV}$, stable within $5 \mathrm{mV}$ for at least $30 \mathrm{~min}$, were used in experiments. Extracellular unit recordings were performed in standard fashion using 8- to 15-megohm broken-tipped glass pipets filled with $1 \mathrm{~m} \mathrm{NaCl}$. Responses were counted from the face of a storage oscilloscope.

Cell input resistances were calculated by measuring the voltage change resulting from injection of constant hyperpolarizing currents (not exceeding $0.5 \mathrm{nA}$ ) intracellularly via a balanced bridge circuit.

All experiments involving local drug application were performed by pres sure-ejection using a two-channel Picospritzer (General Valve Corporation) and either single or double-barrelled pipets, pulled on a Narishige vertical puller so as to fuse the distal tips. Pipets were filled with GLUT, ASP, NAAG, or $A G_{1}$ all $10 \mathrm{~mm}$ in artificial cerebrospinal fluid. Ejection pressures ranged from 5 to 20 (rarely, 30) psi and durations from 5 to an arbitrary maximum of $300 \mathrm{msec}$. For a given ejection, pressure quantity of drug delivered was linearly related to duration of ejection, but the actual drug concentrations delivered to the extracellular space were not determinable. Pipets were checked under a microscope before each penetration to ensure that delivery from both barrels (drop size) was equal. Barrels and ejection order were routinely varied. An interval of at least $30 \mathrm{sec}$ had to be allowed between successive applications of NAAG because of rapid, reversible response decrement (see "Results"). Rare pressure artifacts were clearly distinguishable from drug action.

Mapping experiments were undertaken using extracellularly recorded responses of $51 \mathrm{Ca} 1$ and $\mathrm{CA} 3$ units to $A G$ and GLUT sequentially applied to sites in $\mathrm{s}$. radiatum and pyramidale. Sensitivity to GLUT or AG was tested at several points on the dendritic tree, as detailed below. In CA1, mapping was attempted for three equidistant points in $s$. radiatum and one point in $s$. pyramidale; in CA3, for one point in s. radiatum, one in mossy fibers, and one in s. pyramidale.

\section{Results}

Excitatory actions. Intracellular recordings were obtained from 34 presumed CA1 pyramidal cells, 20 in response to NAAG, and 14 in response to $A G$. Mean resting potential for these cells was $-62 \pm$ $6 \mathrm{mV}$ (mean $\pm \mathrm{SD}$ ), mean action potential amplitude was $71 \pm 7$ $\mathrm{mV}$, and mean input resistance was $27 \pm 6$ megohms. NAAG was found to excite $15(75 \%)$ and AG, $14(100 \%)$ of cells surveyed. GLUT excited all cells tested.

The typical response of neurons to pressure-ejection of these compounds in the region of the apical dendrites was a brief depolarization. Figure $1 A$ shows responses to low doses (brief ejections) of NAAG and GLUT (at the same site) and an evoked excitatory postsynaptic potential (EPSP) for comparison.

The rise time of the depolarization induced by NAAG was less than that of GLUT, as was the rate of decay; however, at low ejection doses, neither approached the rapid rise time of the EPSP. At higher doses of NAAG (Fig. 1B), the response became very rapid, approaching that of the EPSP, and faster than the GLUT response elicited by corresponding doses at the same site. Figure 1, C (low dose) and $D$ (high dose), show that responses to $A G$ were similarly rapid. No decay phase was observed to be slower than the slowest observed GLUT response.

NAAG-responsive sites were scarce, relative to GLUT-responsive sites, and extensive searching was often required. Since the degree of depolarization produced by NAAG usually remained below the action potential threshold, responses were readily observable with intracellular, but not extracellular, recordings. Once a site was found, the sensitivity to NAAG was on the order of one-tenth that of GLUT. NAAG therefore appeared to show a greater selectivity than GLUT with regard to responsive sites. A few sites were insensitive to $A G$ but sensitive to GLUT; rarely, we encountered sites insensitive to GLUT where AG was strongly active. By comparison of length of pressure ejection needed to evoke a response, relative to that necessary for GLUT (arbitrarily set to 1), the potency of NAAG was found to be 0.05 to 0.2 , and of $A G, 0.25$ to 1.0. We observed no significant change in the potencies or responses to these agents under low-calcium conditions.

We occasionally observed slow, long-term, multiphasic responses to NAAG which were quite replicable. These may have resulted from actions at clusters of neighboring siies (Fig. 2, $E$ and $F$ ). Responses to NAAG and AG were replicable, and stable over periods as long as $2 \mathrm{~h}$. No long-term effects of repeated applications on membrane potential or input resistance were seen. In contrast, we invariably observed a marked reversible decrement in the short-term, depolarizing response to NAAG with repeated application (i.e., at less than 15-sec intervals) to the same site. Fig. $2 A$ shows the response to repetitive applications of NAAG at 1 per sec. A marked decrease in response is evident. This response decrement was observed for neither AG (Fig. 2B) nor GLUT (not shown) at similar frequencies. The decrement was not accompanied by changes in input resistance or membrane potential, nor did it require generation of action potentials.

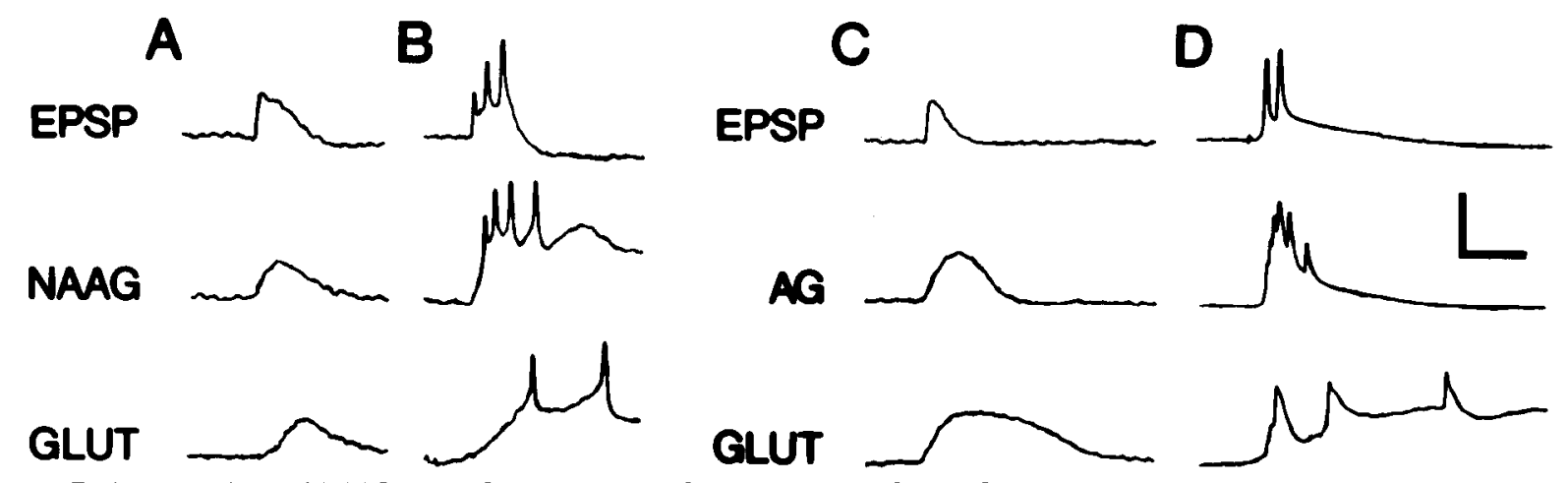

Figure 1. Excitatory actions of NAAG and AG, compared to GLUT and the EPSP. A, Comparison of an evoked EPSP, and response to dendritic application of NAAG (40 msec/20 psi) and GLUT ( $9 \mathrm{msec} / 20 \mathrm{psi})$. Here and below, the cell in question has been hyperpolarized by about $7 \mathrm{mV}$ to better demonstrate the EPSP and agonist responses. This lengthens the decay phase of the EPSP. B. Comparison of an EPSP, NAAG (50/20), and GLIJT (6/20), at a site where these doses produced powerful depolarizations; note speed of rise-time of NAAG. The full decay phases for NAAG and GLUT are not shown. In this and all subsequent figures, action potential amplitudes have been truncated by the pen response of the chart recorder. $C$, Similar comparison of an EPSP with low-dose AG (20/10) and GLUT $(3 / 10)$ applied to the same site. D. Response of the same cell to high doses of AG (10/10) and GLUT (20/ 10). Note very long length of GLUT decay phase in comparison with the EPSP and AG response. calibration: $A$ to $D, 20 \mathrm{mV}, 80 \mathrm{msec}$. 

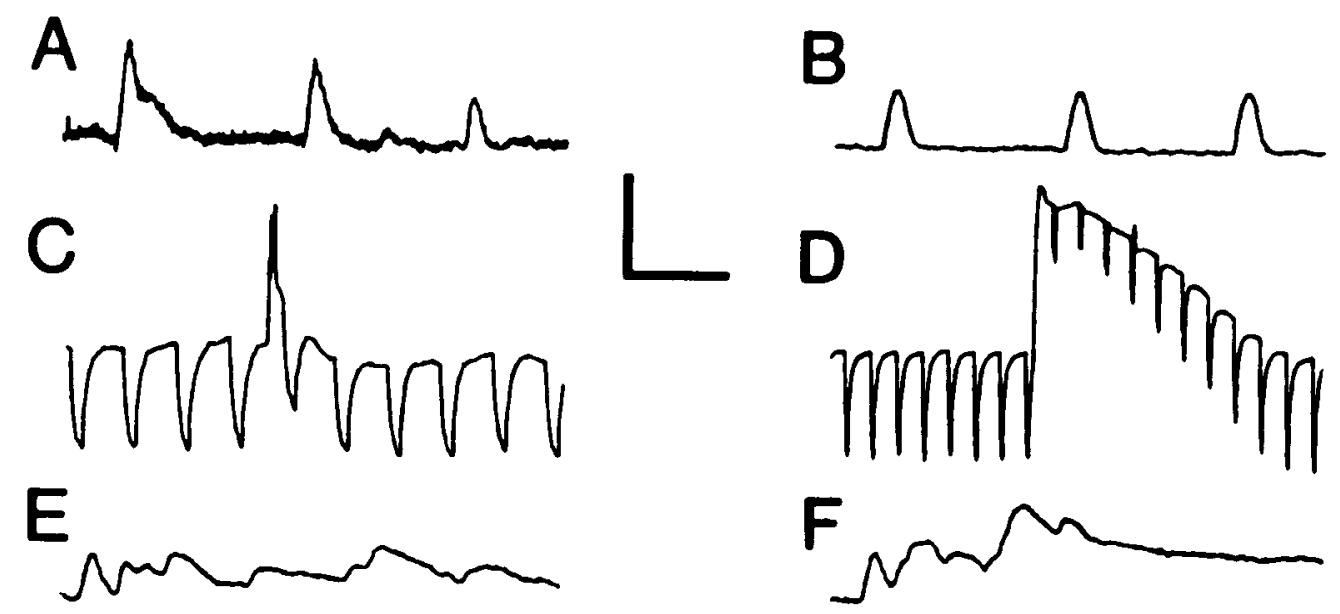

Figure 2. A, Response to repeated ejection of NAAG $(100 \mathrm{msec} / 10 \mathrm{psi})$ results in a decrement. $B$, No decrement occurs in response to repeated $A G(30)$ 20) applications. $C$, Small conductance change in response to dendritic ejection of NAAG (40/20). Downward deflections are hyperpolarizations produced by injections of constant-current $50-\mathrm{msec}$, 0.5-nA pulses through the bridge circuit at a rate of 5 pulses/sec. The fifth current pulse was injected about $50 \mathrm{msec}$ after application of NAAG. $D, A$ large conductance change was observed with AG (150/10). Hyperpolarizing 20-msec, $0.5-\mathrm{nA}$ pulses at $10 / \mathrm{sec}$ were injected through the bridge circuit. The ninth pulse was injected soon after a depolarization produced by dendritic $A G$ application. Note the decrease in voltage step, reflecting an increased conductance after $A G$. The conductance returned to baseline prior to full repolarization. $E$, Example of a prolonged response to NAAG (70/25). F, Response to subsequent application of NAAG in the sarme cell. Note the replicability of the early part of prolonged response. Except for $E$ and $F$, all segments were taken from different experiments. Calibration: $20 \mathrm{mV}, 400 \mathrm{msec}$ for all seg ments.

Low doses of either NAAG or AG produced little change in conductance. In Fig. 2C, a dose of NAAG sufficient to depolarize the cell by $6 \mathrm{mV}$ induced a conductance increase at the soma of small proportions. Large doses, especially of $A G$, produced large conductance increases. In Fig. 2D, hyperpolarizing 20-msec, 0.5-nA pulses at 10 per sec were injected through the bridge circuit. The ninth pulse was injected soon after a depolarization produced by dendritic $A G$ application. Note the decrease in the voltage step, reflecting an increased conductance after $A G$. Conductance returned to baseline prior to full repolarization.

Antagonist effects. In extracellular experiments, bath-applied D, Laminophosphonobutyrate, (D,L-APB; 3 experiments), and D- $\alpha$-aminoadipate, (DAA; 4 experiments), reversibly and replicably decreased the synaptic response to stimulation of the Schaffer-commissural pathway. Minimal antagonist concentrations for blockade of evoked cell firing were $3 \mathrm{~mm}$ for DAA and $5 \mathrm{~mm}$ for D,L-APB (White et al., 1979). At $5 \mathrm{~mm}$ concentrations of each antagonist, there was complete blockade of unit firing evoked by afferent stimulation. Measured at this time, there was a decrement in unit firing to pressure ejection of GLUT to $41 \pm 23 \%$ of control in DAA, and $20 \pm 18 \%$ in APB (expressed as a percentage of the control number of evoked action potentials to GLUT application \pm SE). The corresponding response to AG-application was $45 \pm 12 \%$ of control in DAA and $17 \pm 12 \%$ of control in APB. In three intracellular experiments with $10 \mathrm{mM} \mathrm{D,L} \cdot \mathrm{APB}$, there was complete and reversible suppression of the evoked EPSP and of the response to dendritically applied NAAG and GLUT (10 mM). This action did not depend upon significant depolarization of the cell. Thus, in these experiments, antagonists exerted parallel actions on membrane depolarizations induced by GLUT or AG-application and on the EPSP

Distribution of excitatory sites. Although the difficulty of obtaining NAAG responses made detailed mapping of excitatory profiles impractical, we observed, in three intracellular experiments, that pressure-ejected NAAG evoked either no response (on two occasions) or a hyperpolarizing response (on one occasion) when applied to the cell-body layer. This represented a significant difference from its actions on the dendritic fields, where it evoked depolarizing responses in $75 \%$ of attempted cells.
In multiple mapping experiments, AG appeared significantly more excitatory when applied to the region of the dendritic tree than to the cell soma; in contrast, GLUT and ASP showed no such spatial selectivity. Mapping experiments were performed on a total of 51 units: 40 in CA1 and 11 in CA3. Presumed pyramidal-cell single units were isolated by stepping an extracellular electrode through $\mathrm{s}$. pyramidale, using regular shocks to afferent fibers in $\mathrm{s}$. radiatum as a "hunting stimulus." After a stable unit recording was obtained, a dual-barrel pipet, containing NAAG or AG in one barrel and GLUT in the other, was advanced perpendicular to the plane of the slice through distal, middle, and proximal s. radiatum and then s. pyramidale. We did not examine drug effects in $\mathrm{s}$. oriens. The minimum threshold for drug effect was defined as the smallest drug ejection time required to produce a burst of unit firing. Although "response" was defined formally as a doubling of firing frequency during the 100 -msec period after application of drug, there was in practice never any ambiguity about whether a response did or did not occur. It became evident early in the series that, with occasional exceptions, GLUT exhibited a wider radius of action than did the dipeptides. Consequently, a GLUT response was taken as a signal that the pipet was approaching the unit. At this point, steps were reduced to about $5 \mu \mathrm{m}$, and the NAAG or AG response was evaluated at each point. In a few experiments, AG was used as the initially applied drug and GLUT evaluated second; order of drug ejection had no significant effect, provided 15 - to $30-\mathrm{sec}$ rest periods were interposed. In a given pass of the double pipet, the most sensitive site for each of the amino acids ejected was usually, but not always, the same. Lateral movement (i.e., "off-line") of the pipet by as little as $100 \mu \mathrm{m}$, usually eliminated the response to the amino acids. The data are summarized in Table I and shown graphically in Fig. 3. For both the table and the figure, a smaller number of msec of drug ejection time indicates greater responsiveness to the drug at that site.

In these experiments, on the average, GLUT fired units more readily than did $A G$, requiring $14 \pm 1.4 \mathrm{msec}$ (mean $\pm \mathrm{SE}$ ) of ejection time at the most sensitive site in CAt, versus $52 \pm 10.3 \mathrm{msec}$ for AG. In region $C A 1, A G$ exhibited a markedly greater sensitivity in the dendritic zone than in the somatic zone (see Table I). One-way 
TABLE I

Pressure-ejection of either GLUT or AG needed to evoke unit firing, in various areas of hippocampus and at various points on the dendritic tree and the soma.

One-way ANOVA showed only the spatial profile of $A G$ in $C A 1$ to be statistically significant ( $F$-value, 13.45; $p<0.0001 ; d f=83$ ).

\begin{tabular}{|c|c|c|c|c|}
\hline & AG-CA1 & GLUT-CA1 & AG-CA3 & GLUT-CA3 \\
\hline CELL ${ }^{a}$ & $187 \pm 21.6^{b}(21)^{c}$ & $17 \pm 2.4(18)$ & $252 \pm 35.7(9)$ & $22 \pm 2.3(9)$ \\
\hline $\mathrm{PD}$ & $69 \pm 15.3(21)$ & $19 \pm 3.61(11)$ & ) $214 \pm 32.33$ (12) & 2) $23 \pm 3.46$ (11) \\
\hline MD & $52 \pm 10.26(21)$ & $14 \pm 1.41(18)$ & 3) $275 \pm 51.5(4)$ & $15 \pm 3.00(4)$ \\
\hline $\mathrm{DD}$ & $81 \pm 17.47(21)$ & $17 \pm 1.81(11)$ & 1) $204 \pm 37.39(11)$ & 1) $25 \pm 3.62$ (11) \\
\hline
\end{tabular}

${ }^{a}$ CELL, soma; PD, proximal dendrites; MD, middle dendrites; $D D$, distal dendrites.

${ }^{b}$ Mean \pm SE

'Numbers in parentheses, number of units recorded at each area.

analysis of variance for the $\mathrm{CA} 1$ data for $\mathrm{AG}$ yielded an F-value of $13.45(p<0.0001 ; d f=83)$. GLUT, in contrast, showed no such sensitivity profile $(F$-value $=1.01 ; d f=57 ; p>0.40)$. As a further control, the sensitivity to ASP was compared at cell layer and middendritic regions. No significant difference was found ( $16 \pm 1.9$ msec versus $14 \pm 2.6 \mathrm{msec} ; n=7 ; p>0.40$; paired $t$ test).

Experiments were repeated in CA1 under calcium-free conditions. where both the orthodromically evoked fields and the unit responses were blocked. The threshold ejection time for AG to produce firing was $50.6 \pm 7.7 \mathrm{msec}$ in the dendritic zone versus $156.2 \pm 33.5$ msec in the cell body layer $(n=9)$. One-way analysis of variance for the AG profile in calcium-free perfusate yielded an F-value of $3.45(p<0.04 ; d f=24)$. GLUT ejection-times in calcium-free perfusate failed to show a significant spatial profile (dendritic mean $=14.0 \pm 3.9$ msec; somatic mean $=10.3 \pm 2.0 \mathrm{msec} ; n=9$; $\mathrm{F}$. value $=1.78 ; p>0.18 ; d t=22$ ).

In region CA3, the average sensitivity to GLUT was similar to that observed in CA1, but the average sensitivity to $A G$ was several-fold less. There was no spatial selectivity to the actions of AG in CA3.

\section{Discussion}

We have shown that NAAG and AG have several of the properties expected of an excitatory neurotransmitter in hippocampus. The agents can produce rapid depolarizations and membrane conductance increases when applied to the dendrites of hippocampal pyramidal cells. These depolarizations can be blocked by pharmacological antagonists at mM concentrations. In addition, NAAG and AG show a degree of selectivity in their excitatory actions, in that they are less potent at the cell layer than on the dendritic tree. Each of these properties will be considered in turn.

The depolarizations induced by NAAG and AG showed noteworthy similarities to the endogenous EPSP. The rise and decay times produced by NAAG or AG more closely approximated those of the corresponding EPSP than did those produced by GLUT. NAAG, though of low potency, produced the fastest observed rise times for a given depolarization.

AG produced large conductance increases at high doses, and little or no conductance increase at low doses; such behavior has been noted with many other excitatory amino acids, including GLUT and ASP (Dingledine, 1982; Hablitz, 1982). NAAG usually produced little or no conductance change. This could have been due to its relatively low potency as an agonist, or alternativcly, to a site of action more remote from the soma than those of AG, GLUT, or ASP. Measurement of conductances at the cell soma may misrepresent changes in membrane properties occurring at a remote portion of the dendritic tree.

Previous studies of amino acid antagonists in the Schaffer-commissural pathway (Collingridge, 1983a,b) have demonstrated that high concentrations of antagonists usually are needed to block the endogenous EPSP or the effects of agonists, resulting in low selectivity. In the present experiments, DL-APB and DAA, at the 3 to 10 $\mathrm{mM}$ levels required to block the EPSP, also blocked the action of NAAG, AG, and GLUT. This is a necessary property of a neurotransmitter candidate; yet, in the present experiment, the antagonist effect in CA1 must be considered tairly nonspecific.

Locally applied GLUT was equally excitatory in s. pyramidale and in s. radiatum, despite the paucity of known excitatory afferents to s. pyramidale, and despite the absence of mechanisms for highaffinity GLUT-ASP uptake into s. pyramidale nerve terminals (StormMathisen, 1981; Taxt and Storm-Mathisen, 1984). This makes it probable that, in this area, GLUT was acting at extrajunctional receptors. Such actions of GLUT have been demonstrated previously in binding studies (Foster et al., 1981) and in physiological experiments at the invertebrate neuromuscular junction (Usherwood et al., 1979). NAAG and AG showed markedly less potency at the cell body than on the dendritic tree. Their actions were thus relatively limited to the region of the afferent Schaffer-commissural fibers. In CA3, overall sensitivity to AG appeared to be reduced, and no clear spatial sensitivity profile was observed. Reasons for this are uncertain, since, in fact, some of the afferents to CA3 are shared with CA1. It is possible that the effects of $A G$ application on these common afferents are overshadowed by responses from the mossyfiber afferent system to CA3. The decreased sensitivity could be
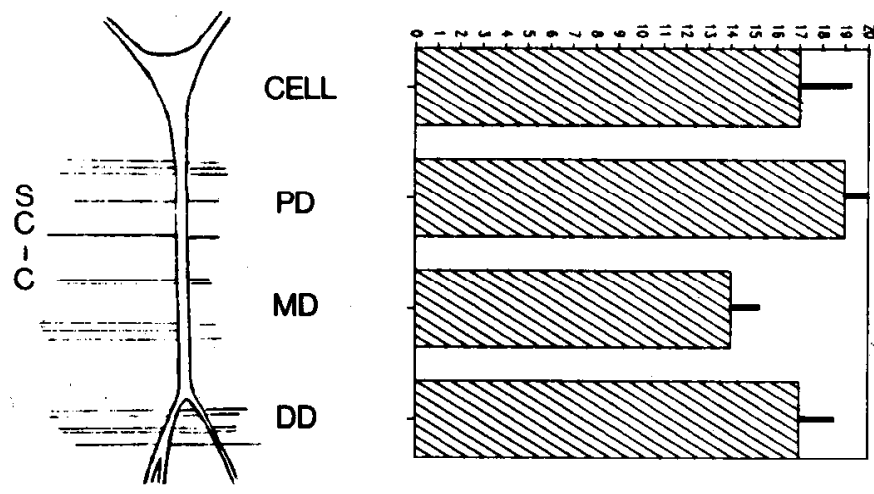

GLUT ms

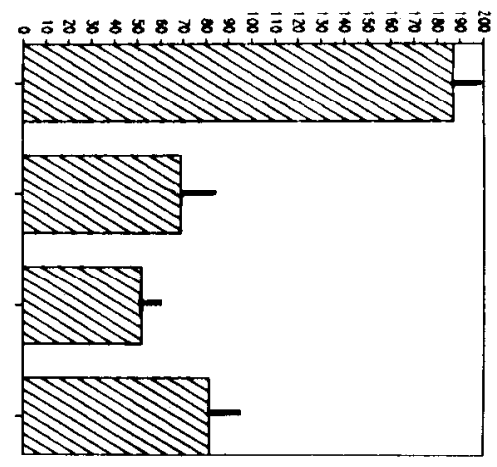

$A G$

Figure 3. The bar graphs show the spatial profile of sensitivity to pressure-ejected GLUT and AG, plotted at sites shown on the idealized CA1 pyramidal cell at left. The first bar graph shows the mean duration of pressure-ejection of GLUT needed to evoke unit firing, at three areas of the dendritic tree, and at the cell soma. Shorter durations reflect greater sensitivity. No significant regional differences in sensitivity to GLUT wore demonstrated by a one-way analysis of variance $(p>0.40, n=18)$. The second bar graph shows the mean duration of pressure-ejection of AG needed to evoke unit firing, at three areas of the dendritic tree, and at the cell soma. Sensitivity to AG is markedly greater in the dendritic, as compared to the somatic, region $(\rho<0.001, n=$ 21, one-way ANOVA). Error bars represent one-half the SE of the mearis. SC-C, Schalfer collateral-comrrissural pathway; $P D$, proximal dendrites; $M D$, middle dendrites; $D D$, distal dendrites. 
physiological or consequent to the greater fragility of CA3 cells and synapses in our experimental paradigm.

This study does not address the actual ionic mechanisms by which AG or NAAG lcads to cell depolarization. The effects of local dipeptide application are similar in low or control-calcium solutions; hence, it is likely that the effects are not strongly dependent upon extracellular calcium nor upon presynaptic factors.

The possibility must be considered that the effects observed here were not due to a direct pharmacological action of the applied amino acids, but to an artifact of application technique. Pressure artifact was usually recognizable when it occurred. Furthermore, GLUT at low ejection pressures often exerted more effect than other substances at high pressures. Last, pressure artifact would not likely have been blocked by standard pharmacological antagonists. Similarly, pH effects were probably not of major importance, since NAAG, the most acidic compound used, consistently gave the weakest excitation. It might be argued that the excitatory actions of NAAG or AG were due to their enzymatic breakdown to GLUT or ASP, but several observations argue against this. First, the distributions of dipeptide-responsive sites and GLUT-responsive sites were not in general the same. Second, the rise and decay times observed with NAAG and AG were often faster than those induced by direct application of GLUT or ASP.

The potencies of NAAG and AG were significantly less than those of GLUT and ASP. This neither affirms nor denies a possible role for the dipeptides as neurotransmitters, since many amino acids, for example, kainic acid or dornoic acid, which are nol endogenous to brain, display high potency when applied locally to cells (Coyle, 1980). The concentrations of neurotransmitter which might obtain at the synaptic cleft in mammalian central nervous system are presently unknown; furthermore, it is difficult to predict what portion of a locally applied drug might arrive in the synapse itself. For these reasons, one can only speculate about the physiological relevance of the excitatory potencies observed with $A G$ and NAAG.

NAAG, but not $A G$, exhibited a striking response decrement with repeated frequent applications. The control response recovered over a few minutes. The mechanism of this effect and its significance are each unclear.

In summary, dipeptides containing equimolar quantities of GLUT and ASP are endogenous to brain, bind avidly to acidic amino acid receptors, and exhibit physiological properties expected of excitatory neurotransmitters in hippocampus. These findings do not prove that NAAG or AG are themselves neurotransmitters; the above properties are necessary, but not sufficient. It appears likely, however, that NAAG and AG represent a family of potentially important excitatory peptides. Further inquiry into the nature of GLUT- and ASP-enriched peptides may yield compounds with even closer identity of action to the endogenous transmitter.

\section{References}

Andersen, P. (1981) Properties of glutamate excitation of hippocampal pyramidal cells. In Glutamate: Transmitter in the Central Nervous System, P. J. Roberts, J. Storm-Mathisen, and G. A. R. Johnston, eds., pp. 25-33, John Wiley and Sons, New York.

Balcar, V. J., and G. A. R. Johnston (1972) The structural specificity of the high affinity uptake of L-glutamate and L-aspartate by rat brain slices. $J$ Neurochem. 19: 2657-2666.

Baudry, M., and G. Lynch (1981) Hippocampal glutamate receptors. Mol. Cell. Biochem. 38: 5-18.

Collingridge G. L., S. J. Kehl, and H. McLennan (1983a) The antagonism of amino-acid induced excitations of rat CA1 hippocampal neurones in vitro. J. Physiol. (Lond.) 334: 19-31.

Collingridge, G. I . S. J. Kehl, and H. Mc.Lennan (1983b) Excitatory amino acids in synaptic transmission in the Schaffer-collateral-commissural pathway of the rat hippocampus. J. Physiol. (Lond.) 334: 33-46.
Cotman, C. W., and J. V. Nadler (1981) Glutamate and aspartate as hippocampal transmitters: biochemical and pharmacological evidence. In Glutamate: Transmitter in the Central Nervous System, P. J. Roberts, J. Storm-Mathisen, and G. A. R. Johnson, eds., pp. 117-154, Wiley and Sons, New York.

Cotman, C. W., A. C. Foster, and T. H. Lanthorn (1981) An overview of glutamate as a neurotransmitter. Adv. Biochem. Psychopharmacol. 27: 128.

Coyle, J. T. (1980) Excitatory amino acid receptors. In Neurotransmitter Receptors, Part 1, S. J. Enna and H. I. Yamamura, eds., pp. 5-40, Chapman and Hall, London.

Curatolo, A., P. d'Archangelo, and A. Lino (1965) Distribution of $N$-acetylaspartic and $\mathrm{N}$-acetyl-aspartyl-glutamic acids in nervous tissue. J. Neurochem. 12: 339-342.

Dingledine, R. (1982) Amino acid-activated calcium conductance in hippocampal pyramidal cells. Soc. Neurosci. Abstr. 8: 796

Fagni, L., M. Baudry, and G. Lynch (1983) Classification and properties of acidic amino acid receptors in hippocampus. J. Neurosci. 3: 1538-1546.

Fonnum, F. (1984) Glutamate: A transmitter in mammalian brain. J. Neurochem. 42: $1-11$.

Foster, A. C., E. E. Mena, G. E. Fagg, and C. W. Cotman (1981) Glutamate and aspartate binding sites are enriched in synaptic junctions isolated from rat brain. J. Neurosci. 1:620-625.

ffrench-Mullen, J. M. H., R. Zaczek, K. Koller, J. T. Coyle, and D. O. Carpenter (1985) N-Acetyl-aspartyl-glutamate: possible role as the neurotransmitter of the lateral olfactory tract. Proc. Natl. Acad. Sci. U. S. A., in press

Hablitz, J. J. (1982) Conductance changes induced by DL-homocysteic acid and $N$-methyl-D-aspartic acid in hippocampal neurons. Brain Res. 247: 149-153.

Koller, K., and J. T. Coyle (1984) Characterization of the interactions of $N$ acetyl-aspartyl-glutamate with $\left[{ }^{3} \mathrm{H}\right]-\mathrm{L}-$ glutamate receptors. Eur. J. Pharmacol. 98: 193-199.

Koller, K., R. Zazcek, and J. T. Coyle (1984) N-Acetyl-aspartyl-glutamate: regional levels in rat brain and effects of brain lesions as determined by a new HPLC method. J. Neurochem. 43: 1136-1142.

Langmoen, I. A., and P. Andersen (1981) The hippocampal slice in vitro. A description of the technique and some of the possibilities it offers. In Electrophysiology of /solated Mammalian CNS Preparations, G. A. Kerkut and H. V. Wheal, eds., pp. 52-105, Academic Press, New York.

Malthe-Sorensson D., K. Skrede, and F. Fonnum (1979) Calcium-dependent release of $\mathrm{D}\left[{ }^{3} \mathrm{H}\right]$-aspartate evoked by selective electrical stimulation of excitatory afferent fibers to hippocampal pyramidal cells in vitro. Neuroscience 4: 1255-1263.

Nadler, J. V., W. F. White, K. W. Vaca, D. A. Redburn, and C. W. Cotman (1976) Aspartate and glutamate as possible transmitters of excitatory hippocampal afferents. Nature 260: 538-540.

Reichert, K. L., and F. Fonnum (1969) Subcellular localization of $N$-acetylaspartyl-glutamate, $\mathrm{N}$-acetyl-glutamate, and glutathione in brain. J. Neurochem. 16: 1409-1416.

Schwartzkroin, P. A., and P. Andersen (1975) Glutamic acid sensitivity of dendrites in hippocampal slices in vitro. Adv. Neurol. 12: 45-51.

Shepherd, G. M. (1979) Hippocampus. In The Synaptic Organization of the Brain, pp. 308-337, Oxford University Press, New York

Storm-Mathisen, J. (1981) Autoradiographic and microchemical localization of high affinity glutamate uptake. In Glutamate: Transmitter in the Central Nervous System, P. J. Roberts, J. Storm-Mathisen, and G. A. R. Johnston, eds., pp. 89-115, John Wiley and Sons, New York.

Taxt, T., and J. Storm-Mathisen (1984) Uptake of D-aspartate and L-glutamate in excitatory axon terminals in hippocampus. Neuroscience 11: 79-100.

Usherwood, P. N. R., R. B. Clark, K. A. Gration, M. Ozeki, and J. Patlak (1979) Glutamatergic synapses and glutamate receptors in locust muscle. J. Physiol. (Paris) 75: 615-621.

White, W. F., J. V. Nadler, and C. W. Cotman (1979) The effect of acidic amino acid antagonists on synaptic transmission in the hippocampal formation in vitro. Brain Res. 164: 177-194.

Wieraszko, A., and G. Lynch (1979) Stimulation-dependent release of possible transmitter substances from hippocampal slices studied with localized perfusion. Brain Res. 160: 372-376.

Zaczek, R., K. Koller, R. Cotter, D. Heller, and J. T. Coyle (1983) N-Acetylaspartyl-glutamate: an endogenous peptide with high affinity for a brain "glutamate" receptor. Proc. Natl. Acad. Sci. U. S. A. 80: 1116-1119. 